TRANSACTIONS OF THE

AMERICAN MATHEMATICAL SOCIETY

Volume 354, Number 10, Pages 3883-3891

S 0002-9947(02)03067-2

Article electronically published on June 4, 2002

\title{
DIFFERENTIAL OPERATORS ON A POLARIZED ABELIAN VARIETY
}

\author{
INDRANIL BISWAS
}

\begin{abstract}
Let $L$ be an ample line bundle over a complex abelian variety $A$. We show that the space of all global sections over $A$ of $\operatorname{Diff}_{A}^{n}(L, L)$ and $S^{n}\left(\operatorname{Diff}_{A}^{1}(L, L)\right)$ are both of dimension one. Using this it is shown that the moduli space $M_{X}$ of rank one holomorphic connections on a compact Riemann surface $X$ does not admit any nonconstant algebraic function. On the other hand, $M_{X}$ is biholomorphic to the moduli space of characters of $X$, which is an affine variety. So $M_{X}$ is algebraically distinct from the character variety if $X$ is of genus at least one.
\end{abstract}

\section{INTRODUCTION}

Let $X$ be a compact connected Riemann surface of genus at least one. A holomorphic connection on a holomorphic line bundle $L$ over $X$ is a first-order differential operator

$$
D: L \longrightarrow K_{X} \otimes L
$$

satisfying the Leibniz rule, which says $D(f s)=f D(s)+\partial f \otimes s$, where $f$ is a locally defined holomorphic function and $s$ is a local holomorphic section of $L$. Let $M_{X}$ denote the moduli space of all rank one holomorphic connections on $X$. In other words, $M_{X}$ parametrizes isomorphism classes of pairs of the form $(L, D)$, where $D$ is a holomorphic connection on $L$. The space $M_{X}$ is a smooth quasi-projective variety of dimension $2 g$, where $g$ is the genus of $X$.

Since any holomorphic connection on a Riemann surface is flat, the monodromy map identifies $M_{X}$ with the character variety $\mathcal{R}:=\operatorname{Hom}\left(\pi_{1}(X), \mathbb{C}^{*}\right)$. This identification is in fact a biholomorphism between $M_{X}$ and $\mathcal{R}$. We show that $\mathcal{R}$ is not algebraically isomorphic to $M_{X}$. More precisely, while $\mathcal{R}$ is an affine variety, $M_{X}$ does not have any nonconstant function (Theorem 3.2).

Let $A$ be a complex abelian variety and $L$ an ample line bundle over $A$. By $\operatorname{Diff}_{A}^{n}(L, L)$ we denote the sheaf of differential operators of order $n$ on $L$.

In Theorem 2.3 we prove that

$$
\operatorname{dim} H^{0}\left(A, S^{n}\left(\operatorname{Diff}_{A}^{1}(L, L)\right)\right)=1
$$

for all $n \geq 1$. As a corollary we have (Corollary 2.10)

$$
\operatorname{dim} H^{0}\left(A, \operatorname{Diff}_{A}^{n}(L, L)\right)=1 .
$$

Theorem 2.3 is the key ingredient also in the proof of Theorem 3.2.

Received by the editors April 5, 2001 and, in revised form, February 5, 2002.

2000 Mathematics Subject Classification. Primary 14K25, 14D20, 14H40.

Key words and phrases. Abelian variety, differential operator, connection, representation space.

(C)2002 American Mathematical Society 
In [6], using the method of the present paper, we prove a Torelli theorem for the moduli space of $\tau$-connections on a compact Riemann surface.

\section{Differential operators AND CONNECtions}

Let $A$ be a complex abelian variety. Fix an ample line bundle $L$ over $A$.

For $n>0$, let $\operatorname{Diff}_{A}^{n}(L, L)$ denote the vector bundle over $A$ defined by the sheaf of differential operators of order $n$ on $L$. So,

$$
\operatorname{Diff}_{A}^{0}(L, L)=\operatorname{Hom}_{\mathcal{O}}(L, L)=\mathcal{O}
$$

and $\operatorname{Diff}_{A}^{n-1}(L, L)$ is a subbundle of $\operatorname{Diff}_{A}^{n}(L, L)$ in an obvious way. More precisely, there is an exact sequence

$$
0 \longrightarrow \operatorname{Diff}_{A}^{n-1}(L, L) \longrightarrow \operatorname{Diff}_{A}^{n}(L, L) \stackrel{\sigma_{n}}{\longrightarrow} S^{n}(T A) \longrightarrow 0
$$

where $\sigma_{n}$ is the symbol homomorphism and $S^{n}(T A)$ is the $n$-th symmetric power of the tangent bundle. By convention, the 0 -th symmetric power of a vector bundle is the trivial line bundle.

The $n$-th symmetric power of the homomorphism $\sigma_{1}$ in (2.1) gives an exact sequence

$$
0 \longrightarrow S^{n-1}\left(\operatorname{Diff}_{A}^{1}(L, L)\right) \longrightarrow S^{n}\left(\operatorname{Diff}_{A}^{1}(L, L)\right) \stackrel{S^{n}\left(\sigma_{1}\right)}{\longrightarrow} S^{n}(T A) \longrightarrow 0
$$

of vector bundles. The vector bundle $S^{n-1}\left(\operatorname{Diff}_{A}^{1}(L, L)\right)$ is realized as a subbundle using the composition

$$
S^{n-1}\left(\operatorname{Diff}_{A}^{1}(L, L)\right) \stackrel{\alpha}{\longrightarrow} S^{n-1}\left(\operatorname{Diff}_{A}^{1}(L, L)\right) \otimes \operatorname{Diff}_{A}^{1}(L, L) \stackrel{\beta}{\longrightarrow} S^{n}\left(\operatorname{Diff}_{A}^{1}(L, L)\right),
$$

where $\alpha$ is defined using the inclusion of $\mathcal{O}$ in $\operatorname{Diff}_{A}^{1}(L, L)$ in the exact sequence (2.1) and $\beta$ is the symmetrization.

Theorem 2.3. For $n \geq 1$, the homomorphism

$$
H^{0}\left(A, S^{0}\left(\operatorname{Diff}_{A}^{1}(L, L)\right)\right)=H^{0}(A, \mathcal{O}) \longrightarrow H^{0}\left(A, S^{n}\left(\operatorname{Diff}_{A}^{1}(L, L)\right)\right)
$$

obtained using (2.2) repeatedly is an isomorphism.

Proof. Consider the long exact sequence of cohomologies

$$
\begin{gathered}
H^{0}\left(A, S^{n-1}\left(\operatorname{Diff}_{A}^{1}(L, L)\right)\right) \longrightarrow H^{0}\left(A, S^{n}\left(\operatorname{Diff}_{A}^{1}(L, L)\right)\right) \\
\longrightarrow H^{0}\left(A, S^{n}(T A)\right) \stackrel{h_{n}}{\longrightarrow} H^{1}\left(A, S^{n-1}\left(\operatorname{Diff}_{A}^{1}(L, L)\right)\right)
\end{gathered}
$$

obtained from (2.2). To prove the theorem, it suffices to show that the above homomorphism $h_{n}$ is injective for all $n \geq 1$. Indeed, if $h_{n}$ is injective, then the injective map

$$
H^{0}\left(A, S^{n-1}\left(\operatorname{Diff}_{A}^{1}(L, L)\right)\right) \longrightarrow H^{0}\left(A, S^{n}\left(\operatorname{Diff}_{A}^{1}(L, L)\right)\right)
$$

is also surjective.

A connected homomorphism, like $h_{n}$ in (2.4), is the cup product by the extension class for the corresponding short exact sequence. So we need to understand the extension class

$$
\bar{C}_{n} \in H^{1}\left(A, \operatorname{Hom}\left(S^{n}(T A), S^{n-1}\left(\operatorname{Diff}_{A}^{1}(L, L)\right)\right)\right)
$$


for the exact sequence (2.2). Using the homomorphism $S^{n-1}\left(\sigma_{1}\right)$ in $(2.2)$, the cohomology class $\bar{C}_{n}$ gives

$$
C_{n} \in H^{1}\left(A, \operatorname{Hom}\left(S^{n}(T A), S^{n-1}(T A)\right)\right) .
$$

This cohomology class $C_{n}$ is clearly the extension class for the exact sequence

$$
\begin{aligned}
& 0 \longrightarrow S^{n-1}\left(\operatorname{Diff}_{A}^{1}(L, L)\right) / S^{n-2}\left(\operatorname{Diff}_{A}^{1}(L, L)\right)=S^{n-1}(T A) \\
& \longrightarrow S^{n}\left(\operatorname{Diff}_{A}^{1}(L, L)\right) / S^{n-2}\left(\operatorname{Diff}_{A}^{1}(L, L)\right) \stackrel{S^{n}\left(\sigma_{1}\right)}{\longrightarrow} S^{n}(T A) \longrightarrow 0
\end{aligned}
$$

obtained from (2.2). Before we describe $C_{n}$, we need to identify the extension class for the exact sequence from which (2.2) is built, namely, the one obtained by setting $n=1$ in (2.1).

The first step would be to show that the extension class for the exact sequence

$$
0 \longrightarrow \mathcal{O} \longrightarrow \operatorname{Diff}_{A}^{1}(L, L) \stackrel{\sigma_{1}}{\longrightarrow} T A \longrightarrow 0
$$

is the first Chern class of $L$. Although this fact is well known, we give brief details of the argument.

We fix a convention. For our convenience, the first Chern class will always denote $2 \pi \sqrt{-1}$ times the standard rational class. So, for example, $c_{1}(L) \in$ $H^{2}(J, 2 \pi \sqrt{-1} \mathbb{Z})$.

Let $\left\{U_{i}\right\}_{i \in I}$ be a covering of $A$ by analytic open sets and

$$
\phi_{i}:\left.L\right|_{U_{i}} \longrightarrow \mathcal{O}_{U_{i}}
$$

be local trivializations of $L$. The composition $\phi_{j} \circ\left(\phi_{i}\right)^{-1}$ is a multiplication by a function on $U_{i} \cap U_{j}$. This function will be denoted by $\phi_{i, j}$.

Using $\phi_{i}$ and the differentiation action of $T U_{i}$ on $\mathcal{O}_{U_{i}}$, we have a splitting

$$
\psi_{i}: T U_{i} \longrightarrow \operatorname{Diff}_{U_{i}}^{1}\left(\left.L\right|_{U_{i}},\left.L\right|_{U_{i}}\right)
$$

of the symbol map. The difference $\psi_{j}-\psi_{i}$ on $U_{i} \cap U_{j}$ factors as a composition homomorphism

$$
T\left(U_{i} \cap U_{j}\right) \stackrel{\gamma}{\longrightarrow} \mathcal{O}_{U_{i} \cap U_{j}} \hookrightarrow \operatorname{Diff}_{U_{i} \cap U_{j}}^{1}\left(\left.L\right|_{U_{i} \cap U_{j}},\left.L\right|_{U_{i} \cap U_{j}}\right),
$$

and the one-form $\gamma$ on $U_{i} \cap U_{j}$ coincides with $d \phi_{i, j} / \phi_{i, j}$. Therefore, the one-cocyle $\left\{d \phi_{i, j} / \phi_{i, j}\right\}_{i, j \in I}$ represents the extension class in $H^{1}\left(A, \Omega_{A}^{1}\right)$ for the exact sequence (2.7). On the other hand, $\left\{d \phi_{i, j} / \phi_{i, j}\right\}$ represents the Chern class $c_{1}(L)$.

Since the exact sequence (2.2) is simply the $n$-th symmetric power of (2.7), the extension class $C_{n}$ in (2.5) is also $c_{1}(L)$. To explain this, first note that the cup product of $c_{1}(L) \in H^{1}\left(A, \Omega_{A}^{1}\right)$ with the identity automorphism of $S^{n}(T A)$ is a cohomology class

$$
c \in H^{1}\left(A, \operatorname{Hom}\left(S^{n}(T A), \Omega_{A}^{1} \otimes S^{n}(T A)\right)\right) .
$$

Using the contraction $\Omega_{A}^{1} \otimes S^{n}(T A) \longrightarrow S^{n-1}(T A)$, the cohomology class $c$ gives

$$
C_{n}^{\prime} \in H^{1}\left(A, \operatorname{Hom}\left(S^{n}(T A), S^{n-1}(T A)\right)\right) .
$$

The extension class $C_{n}$ in (2.5) coincides with $C_{n}^{\prime}$. Indeed, since the extension class for $(2.7)$ is $c_{1}(L)$, this is an immediate consequence of the fact that $(2.2)$ is the symmetric power of (2.7).

Take a translation invariant $(1,1)$-form $\omega$ on the abelian variety $A$ such that $\omega$ represents the first Chern class $c_{1}(L)$. It is easy to see that there is exactly one 
such form. Since $L$ is ample, the form $\omega$ must be positive. In other words, the homomorphism

$$
\widehat{\omega}: T A \longrightarrow \Omega_{A}^{0,1}
$$

that sends any $v \in T_{p} A$ to the contraction of $\omega(p)$ with $v$ is an isomorphism.

Since $T A$ is trivial, any section of $S^{n}(T A)$ is invariant under translations in $A$. Take a nonzero section

$$
0 \neq \xi \in H^{0}\left(A, S^{n}(T A)\right) .
$$

Using the contraction map $\widehat{\omega}$ in $(2.8)$, the section $\xi$ gives a $(0,1)$-form

$$
\bar{\xi} \in \Omega^{0,1}\left(S^{n-1}(T A)\right)
$$

with values in $S^{n-1}(T A)$. We noted earlier that $C_{n}$ in (2.5) coincides with $C_{n}^{\prime}$. Therefore, the $S^{n-1}(T A)$-valued $(0,1)$-form $\bar{\xi}$ represents the cohomology class

$$
\bar{S}^{n-1}\left(\sigma_{1}\right) \circ h_{n}(\xi) \in H^{1}\left(A, S^{n-1}(T A)\right)
$$

in Dolbeault cohomology, where $h_{n}$ is the connecting homomorphism in (2.4) and

$$
\bar{S}^{n-1}\left(\sigma_{1}\right): H^{1}\left(A, S^{n-1}\left(\operatorname{Diff}_{A}^{1}(L, L)\right)\right) \longrightarrow H^{1}\left(A, S^{n-1}(T A)\right)
$$

is the homomorphism obtained, in an obvious fashion, from $S^{n-1}\left(\sigma_{1}\right)$ in (2.2).

Since both $\omega$ and $\xi$ are invariant under the translations in $A$, the form $\bar{\xi}$ is also invariant under the translations. Furthermore, since $\widehat{\omega}$ in $(2.8)$ is an isomorphism and $\xi \neq 0$, we have $\bar{\xi} \neq 0$. From this it follows that the cohomology class in $H^{1}\left(A, S^{n-1}(T A)\right)$ represented by $\bar{\xi}$ is nonzero. To see this, note that $\omega$ being positive defines a Kähler structure on $A$. In order to prove that the cohomology class in $H^{1}\left(A, S^{n-1}(T A)\right)$ represented by $\bar{\xi}$ is nonzero, it suffices to show that the form $\bar{\xi}$ is harmonic for the Dolbeault complex for $S^{n-1}(T A)$. However, since the Kähler form is translation invariant, $\bar{\xi}$ being translation invariant must be harmonic.

We already noted that the Dolbeault cohomology class represented by $\bar{\xi}$ coincides with $\bar{S}^{n-1}\left(\sigma_{1}\right) \circ h_{n}(\xi)$. Since this class is nonzero, $h_{n}(\xi)$ must be nonzero. In other words, the homomorphism $h_{n}$ in (2.4) is injective. We noted earlier that the injectivity of $h_{n}$ proves the theorem. Therefore, the proof of the theorem is complete.

For $n \geq 1$, consider the exact sequence

$$
\begin{aligned}
& 0 \longrightarrow \operatorname{Diff}_{A}^{n-1}(L, L) / \operatorname{Diff}_{A}^{n-2}(L, L)=S^{n-1}(T A) \\
& \longrightarrow \operatorname{Diff}_{A}^{n}(L, L) / \operatorname{Diff}_{A}^{n-2}(L, L) \stackrel{\sigma_{n}}{\longrightarrow} S^{n}(T A) \longrightarrow 0
\end{aligned}
$$

obtained from (2.1), where $\operatorname{Diff}_{A}^{-1}(L, L)$ denotes 0 . It is known that the exact sequence (2.8) is isomorphic to the exact sequence (2.6). Therefore, the injectivity of the homomorphism $h_{n}$ in (2.4) implies that the connecting homomorphism

$$
H^{0}\left(A, S^{n}(T A)\right) \longrightarrow H^{1}\left(A, S^{n-1}(T A)\right)
$$

in the long exact sequence of cohomologies for (2.9) is also injective. Consequently, the injective homomorphism

$$
H^{0}\left(A, \operatorname{Diff}_{A}^{n-1}(L, L)\right) \longrightarrow H^{0}\left(A, \operatorname{Diff}_{A}^{n}(L, L)\right)
$$

obtained from (2.1) is also surjective. Therefore, we have the following corollary of Theorem 2.3. 
Corollary 2.10. The inclusion

$$
H^{0}(A, \mathcal{O}) \longrightarrow H^{0}\left(A, \operatorname{Diff}_{A}^{n}(L, L)\right)
$$

obtained from (2.1) is an isomorphism for all $n \geq 0$.

Consider the exact sequence

$$
0 \longrightarrow \Omega_{A}^{1} \longrightarrow \operatorname{Diff}_{A}^{1}(L, L)^{*} \stackrel{\tau}{\longrightarrow} \mathcal{O} \longrightarrow 0,
$$

which is the dual of (2.7). We will denote by $\overline{1}$ the image of the section of $\mathcal{O}$ defined by the constant function 1 . The subset of the total space of the vector bundle $\operatorname{Diff}_{A}^{1}(L, L)^{*}$ defined by the inverse image $\tau^{-1}(\overline{1})$ will be denoted by $\mathcal{C}(L)$.

Let

$$
p: \mathcal{C}(L) \longrightarrow A
$$

be the obvious projection. The exact sequence (2.11) shows that for any point $x \in A$, the inverse image $p^{-1}(x)$ is an affine space for the holomorphic cotangent space $\left(\Omega_{A}^{1}\right)_{x}$.

Let $U \subset A$ be an open subset and $\theta$ a holomorphic section over $U$ of the fiber bundle $\mathcal{C}(L)$. Such a section $\theta$ defines a holomorphic connection on $\left.L\right|_{U}$ [1]. The exact sequence (2.7) for a holomorphic line bundle over a complex manifold is known as the Atiyah exact sequence. A splitting of the Atiyah exact sequence is a holomorphic connection [1]. A section $\theta$ of $\mathcal{C}(L)$ over $U$ clearly gives a splitting over $U$ of the exact sequence $(2.7)$.

The subset $\mathcal{C}(L) \subset \operatorname{Diff}_{A}^{1}(L, L)^{*}$ being a Zariski open set has a natural algebraic structure. By $\mathcal{O}_{\mathcal{C}(L)}$ we will denote the structure sheaf of this algebraic variety.

Proposition 2.13. For the variety $\mathcal{C}(L)$,

$$
H^{0}\left(\mathcal{C}(L), \mathcal{O}_{\mathcal{C}(L)}\right)=\mathbb{C}
$$

or, in other words, there is no nonconstant algebraic function on $\mathcal{C}(L)$.

Proof. Let $P:=P \operatorname{Diff}_{A}^{1}(L, L)^{*}$ be the projective bundle over $A$ consisting of lines in $\operatorname{Diff}_{A}^{1}(L, L)^{*}$. Similarly, $P^{\prime}:=P \Omega_{A}^{1}$ denotes the projective bundle defined by the lines in $\Omega_{A}^{1}$. Using the inclusion of $\Omega_{A}^{1}$ in $\operatorname{Diff}_{A}^{1}(L, L)^{*}$ in $(2.11)$, we have $P^{\prime}$ as a subbundle of the projective bundle $P$. Let

$$
P_{0}:=P-P^{\prime}
$$

be the complement. It is easy to see that $P_{0}$ is naturally identified with $\mathcal{C}(L)$. The identification is defined by the obvious projection to $P$ of the complement of the zero section in $\operatorname{Diff}_{A}^{1}(L, L)^{*}$.

Since the quotient bundle $\operatorname{Diff}_{A}^{1}(L, L)^{*} / \Omega_{A}^{1}$ is trivial, the divisor $P^{\prime}$ on $P$ is the divisor of the tautological line bundle $\mathcal{O}_{P}(1)$ over $P$. So a meromorphic function on $P$ with pole of order $d$ along $P^{\prime}$ is a section of $\mathcal{O}_{P}(d)$. Therefore, it suffices to prove that

for all $d \geq 0$.

$$
\operatorname{dim} H^{0}\left(P, \mathcal{O}_{P}(d)\right)=1
$$

Let $\gamma$ denote the projection of $P$ to $A$. Taking direct image to $A$, we have the identification

$$
H^{0}\left(P, \mathcal{O}_{P}(d)\right)=H^{0}\left(A, \gamma_{*} \mathcal{O}_{P}(d)\right)=H^{0}\left(A, S^{d}\left(\operatorname{Difff}_{A}^{1}(L, L)\right)\right) .
$$

Now Theorem 2.3 implies that $\operatorname{dim} H^{0}\left(P, \mathcal{O}_{P}(d)\right)=1$ for $d \geq 0$. This completes the proof of the proposition. 
In the next section we will specialize to Jacobians of curves.

\section{RANK OnE CONNECTIONS ON A CURVE}

Let $X$ be a connected smooth projective curve over $\mathbb{C}$ or, equivalently, a compact connected Riemann surface. The genus $g$ of $X$ is assumed to be positive. Fix once and for all a point $x_{0} \in X$. Let $J:=\operatorname{Pic}^{0}(X)$ be the Jacobian of $X$. We will denote by $\Theta$ the line bundle over $J$ defined by the divisor that consists of all $L$ with

$$
H^{0}\left(X, \mathcal{O}_{X}\left((g-1) x_{0}\right) \otimes L\right) \neq 0 .
$$

It is known that $\Theta$ is ample. More precisely, it defines a principal polarization on $J$.

Let $M_{X}$ denote the moduli space of rank one holomorphic connections on $X$. In other words, $M_{X}$ parametrizes pairs of the form $(L, D)$, where $L$ is a holomorphic line bundle over $X$ and $D$ is a holomorphic connection on $L$. Since $\operatorname{dim} X=1$, any holomorphic connection on $X$ is flat. The moduli space of holomorphic connections on a smooth projective variety has been constructed in $\left[5\right.$. In particular, $M_{X}$ is a quasi-projective variety.

Let

$$
\phi: M_{X} \longrightarrow J
$$

be the forgetful morphism. So $\phi$ sends a pair $(L, D)$ to $L$.

Let $\mathcal{R}$ denote the character variety $\operatorname{Hom}\left(\pi_{1}(X), \mathbb{C}^{*}\right)$ of the fundamental group. If we fix generators of the fundamental group $\pi_{1}(X)$, then $\mathcal{R}$ gets identified with the $2 g$-fold self-product $\mathbb{C}^{*} \times \mathbb{C}^{*} \times \cdots \times \mathbb{C}^{*}$.

By associating its monodromy to a flat connection, the space $\mathcal{R}$ gets identified with $M_{X}$. More precisely, for $D \in M_{X}$, this identification associates $D$ with the element in $\mathcal{R}$ that sends any $g \in \pi_{1}(X)$ to the holonomy of $D$ around $g$. This identification of $M_{X}$ with $\mathcal{R}$ is biholomorphic but not necessarily algebraic [5. In fact, we will see that $M_{X}$ is not algebraically isomorphic to $\mathcal{R}$.

Since $\mathcal{R}$ is a product of copies of $\mathbb{C}^{*}$, it is an affine variety. In particular, there are many nonconstant functions on $\mathcal{R}$. In view of that, the following theorem shows that $M_{X}$ is not algebraically isomorphic to $\mathcal{R}$.

Theorem 3.2. For the variety $M_{X}$,

$$
\operatorname{dim} H^{0}\left(M_{X}, \mathcal{O}_{M_{X}}\right)=1,
$$

where $\mathcal{O}_{M_{X}}$ denotes the structure sheaf.

Proof. Set the pair $(A, L)$ is Section 2 to be $(J, \Theta)$. Consider the fiber bundle

$$
p: \mathcal{C}(\Theta) \longrightarrow J
$$

constructed in (2.12). In view of Proposition 2.13, the theorem follows immediately from the following proposition.

Proposition 3.3. The fiber bundle $\mathcal{C}(\Theta)$ over $J$ defined by $p$ is algebraically isomorphic to $M_{X}$ defined in (3.1).

Proof. We already remarked that $\mathcal{C}(\Theta)$ is an affine bundle over $J$ for the cotangent bundle, that is, any fiber of $p$ is an affine space for the cotangent space at that point. Now note that $M_{X}$ is also an affine bundle for the cotangent bundle. Indeed, the space of holomorphic connections on a degree zero line bundle over $X$ is an affine 
space for $H^{0}\left(X, K_{X}\right)$, where $K_{X}$ denotes the holomorphic cotangent bundle. On the other hand, $H^{0}\left(X, K_{X}\right)$ are the fibers $\Omega_{J}^{1}$.

Affine bundles for the cotangent bundle are classified by $H^{1}\left(J, \Omega_{J}^{1}\right)$. We will quickly recall how a cohomology class is associated to an affine bundle.

Let $q: Z \longrightarrow J$ be an affine bundle for $\Omega_{J}^{1}$. Let $\left\{U_{i}\right\}_{i \in I}$ be a covering of $J$ by analytic open subsets and

$$
\psi_{i}:\left.U_{i} \longrightarrow Z\right|_{U_{i}}
$$

holomorphic sections. Since the fibers of $Z$ are affine spaces, $\psi_{j}-\psi_{i}$ is a holomorphic section of $\Omega_{U_{i} \cap U_{j}}^{1}$. These one-forms $\left\{\psi_{j}-\psi_{i}\right\}_{i, j \in I}$ define a cocyle. Let $\beta_{Z} \in H^{1}\left(J, \Omega_{J}^{1}\right)$ be the corresponding cohomology class. It is easy to see that another affine bundle $Z^{\prime}$ will be holomorphically isomorphic to $Z$ if $\beta_{Z}$ coincides with the corresponding cohomology class $\beta_{Z^{\prime}}$ for $Z^{\prime}$. If these two affine bundles are analytically isomorphic, then from the GAGA principle of [4], it follows that they must be algebraically isomorphic.

If $\beta_{Z} \neq 0$ and $\beta_{Z^{\prime}}=\lambda \beta_{Z}$, where $\lambda \in \mathbb{C}^{*}$, then also the two fiber bundles $Z$ and $Z^{\prime}$ are algebraically isomorphic. However, if $\lambda \neq 1$, then there will be no isomorphism preserving the affine space structures. Nevertheless, there will be an isomorphism $h: Z^{\prime} \longrightarrow Z$ of fiber bundles satisfying the identity $h(z+\theta)=$ $h(z)+\lambda \theta$, where $\theta \in \Omega_{J}^{1}$.

Let $\beta_{p}$ (respectively, $\beta_{\phi}$ ) be the cohomology class in $H^{1}\left(J, \Omega_{J}^{1}\right)$ associated to $\mathcal{C}(\Theta)$ (respectively, $M_{X}$ ). We will show that both $\beta_{p}$ and $2 \beta_{\phi}$ coincide with $c_{1}(\Theta)$.

In the proof of Theorem 2.3, we have seen that the extension class for the Atiyah exact sequence $(2.7)$ for $\Theta$ coincides with $c_{1}(\Theta)$. We already noted that any section $\psi:\left.U \longrightarrow \mathcal{C}(\Theta)\right|_{U}$ as in (3.4) gives a splitting over $U$ of the Atiyah exact sequence for $\Theta$. Consequently, $\beta_{p}$ coincides with $c_{1}(\Theta)$.

Let

$$
f: J \longrightarrow M_{X}
$$

be a $C^{\infty}$ section of the map $\phi$ in (3.1). The obstruction to the holomorphicity of the map $f$ gives a form $\omega_{f}$ on $J$ of type $(1,1)$. This form $\omega_{f}$ can be described as follows. For any point $z \in J$, let

$$
d f(z): T_{z}^{\mathbb{R}} J \longrightarrow T_{f(z)}^{\mathbb{R}} M_{X}
$$

be the homomorphism of real tangent spaces given by the differential of $f$. Let

$$
J_{z}: T_{z}^{\mathbb{R}} J \longrightarrow T_{z}^{\mathbb{R}} J
$$

be the almost complex structure of $J$ at $z$. Similarly, the almost complex structure of $M_{X}$ at $f(z)$ will be denoted by $J_{f(z)}$. Now, for any $v \in T_{z}^{\mathbb{R}} J$, the difference

$$
J_{f(z)} \circ d f(z)(v)-d f(z) \circ J_{z}(v)
$$

is an element of $\left(T_{z}^{\mathbb{R}} J\right)^{*}$. Indeed, this is an immediate consequence of the fact that the kernel of the differential homomorphism

$$
d \phi(f(z)): T_{f(z)}^{\mathbb{R}} M_{X} \longrightarrow T_{z}^{\mathbb{R}} J
$$

is identified with $\left(T_{z}^{\mathbb{R}} J\right)^{*}$ using the affine space structure of the fibers of $\phi$. The resulting homomorphism $T_{z}^{\mathbb{R}} J \longrightarrow\left(T_{z}^{\mathbb{R}} J\right)^{*}$ that sends any $v$ to $J_{f(z)} \circ d f(z)(v)-$ $d f(z) \circ J_{z}(v)$ defines the $(1,1)$-form $\omega_{f}$.

The cohomology class in $H^{1}\left(J, \Omega_{J}^{1}\right)$ represented by $\omega_{f}$ coincides with $\beta_{\phi}$. In fact, this is the Dolbeault analog of the earlier construction of the cohomology class $\beta_{Z}$. 
Any holomorphic line bundle over $X$ of degree zero admits a unique unitary flat connection. Let $f$ be the map that associates to any $L$ in $J$ the unitary flat connection on $L$. From [2, Theorem 2.11] we know that $2 \omega_{f}$ coincides with the pullback, using $f$, of a certain natural symplectic form on $M_{X}$. The symplectic form on $M_{X}$ in question is the one defined in [3] on the representation space $\mathcal{R}$. On the other hand, the pullback of this symplectic form coincides with $c_{1}(\Theta)$. This is well known; the details can be found in [2].

Therefore, both $\beta_{p}$ and $2 \beta_{\phi}$ coincide with $c_{1}(\Theta)$. This completes the proof of the proposition.

We already noted that Proposition 3.3 completes the proof of Theorem 3.2. Therefore, the proof of Theorem 3.2 is complete.

Let $Y$ be another compact connected Riemann surface. Let $M_{Y}$ denote the moduli space of rank one holomorphic connections on $Y$. Let $\Omega(X)$ (respectively, $\Omega(Y)$ ) denote the natural symplectic form on $M_{X}$ (respectively, $M_{Y}$ ) constructed in 3 .

Proposition 3.5. If there is an algebraic isomorphism of $M_{X}$ with $M_{Y}$ that takes the symplectic form $\Omega(X)$ to $\Omega(Y)$, then the Riemann surface $X$ is isomorphic to $Y$.

Proof. The Torelli theorem says that if the Jacobian of $X$ is isomorphic to the Jacobian of $Y$ as a principally polarized abelian variety, then $X$ is isomorphic to $Y$. The principal polarization in question is the one given by theta. The proposition will be proved by recovering the Jacobian of $X$, along with its polarization, from the symplectic variety $\left(M_{X}, \Omega(X)\right)$.

Let $\phi_{Y}: M_{Y} \longrightarrow \operatorname{Pic}^{0}(Y)$ be the projection defined in (3.1) for $Y$.

There is no nonconstant algebraic map from the affine line to an abelian variety. This is an immediate consequence of the fact that there is no nonzero holomorphic one-form on the projective line. Therefore, any algebraic isomorphism

$$
\psi: M_{X} \longrightarrow M_{Y}
$$

induces an isomorphism $\bar{\psi}: \operatorname{Pic}^{0}(X) \longrightarrow \operatorname{Pic}^{0}(Y)$ which is determined by the identity

$$
\phi_{Y} \circ \psi=\bar{\psi} \circ \phi
$$

where $\phi$ is as in (3.1). Consequently, $M_{X}$ determines both $\operatorname{Pic}^{0}(X)$ and the projection $\phi$.

Take a $C^{\infty}$ section

$$
f: \operatorname{Pic}^{0}(X) \longrightarrow M_{X}
$$

(as in the proof of Proposition 3.3) of the projection $\psi$. As in the proof of Proposition 3.3 , let $\omega_{f}$ denote the $(1,1)$-form on $\operatorname{Pic}^{0}(X)$ given by the obstruction to the holomorphicity of $f$. If

$$
f_{0}: \operatorname{Pic}^{0}(X) \longrightarrow M_{X}
$$

is another section of $\psi$, then it is easy to check that

$$
\omega_{f}-\omega_{f_{0}}=\bar{\partial}\left(f-f_{0}\right) \text {. }
$$

Note that using the affine bundle structure of $M_{X}$, the difference $f-f_{0}$ defines a $(1,0)$-form on $\operatorname{Pic}^{0}(X)$.

Set $f_{0}$ to be the section that sends any line bundle $L$ in $\operatorname{Pic}^{0}(X)$ to the (unique) unitary flat connection on $L$. In the proof of Proposition 3.3 we saw that $\omega_{f_{0}}$ 
represents $c_{1}(\Theta) / 2$. Therefore, the identity (3.6) implies that the cohomology class in $H^{1}\left(\mathrm{Pic}^{0}(X), \Omega_{\mathrm{Pic}^{0}(X)}^{1}\right)$ represented by the form $2 \omega_{f}$ coincides with $c_{1}(\Theta)$.

Therefore, the algebraic variety $M_{X}$ equipped with the symplectic form $\Omega(X)$ determines the principally polarized abelian variety $\left(\operatorname{Pic}^{0}(X), c_{1}(\Theta)\right)$. This completes the proof of the proposition.

Since only the cohomology class represented by the symplectic form is used, $X$ is isomorphic to $Y$ if there is an isomorphism of $M_{X}$ with $M_{Y}$ that takes the cohomolgy class for the symplectic form $\Omega(Y)$ to that for $\Omega(X)$.

\section{REFERENCES}

[1] M.F. Atiyah, Complex analytic connections in fibre bundles, Trans. Amer. Math. Soc. 85 (1957), 181-207. MR 19:172c

[2] I. Biswas and N. Raghavendra, Curvature of the determinant bundle and the Kähler form over the moduli of parabolic bundles for a family of pointed curves, Asian Jour. Math. 2 (1998), 303-324. MR 99k:32032

[3] W.M. Goldman, The symplectic nature of fundamental groups of surfaces, Adv. in Math. 54 (1984), 200-225. MR 86i:32042

[4] J.-P. Serre, Géométrie algébrique et géométrie analytique, Ann. Inst. Fourier Grenoble 6 (1955), 1-42. MR 18:511a

[5] C.T. Simpson, Moduli of representations of the fundamental group of a smooth projective variety. II, Inst. Hautes Études Sci. Publ. Math. 80 (1994), 5-79. MR 96e:14013

[6] I. Biswas, On the moduli space of $\tau$-connections on a Riemann surface, preprint (2001).

School of Mathematics, Tata Institute of Fundamental Research, Homi Bhabha RoAd, Bombay 400005, India

E-mail address: indranil@math.tifr.res.in 